\section{Optical Thermal Cycler for Use as a Fluorimetric Plate Reader to Estimate DNA Concentrations}

BioTechniques 34:710-712 (April 2003)

Recent developments in PCR techniques provide the opportunity to quantify low copy numbers of specific nucleic acid sequences by quantitative, real-time PCR (1). The use of quantitative, real-time PCR is spreading due to its accuracy and relative simplicity. As a consequence, the number of laboratories equipped with an optical thermal cycler is increasing. The use of an apparatus for more than one single purpose is desirable because this would eliminate the need for purchasing costly extra equipment. For instance, optical thermal cyclers are also being used to perform melting curves in SNP differentiation (2), PCR product differentiation (3), and $\mathrm{G}+\mathrm{C}$ mol \% content estimates in microorganisms (4). Here we suggest a nonstandard use of an optical thermal cycler as a fluorescent plate reader and its application to the estimation of nucleic acid concentrations. This is achieved by using a dsDNA-specific fluorescent dye, SYBR ${ }^{\circledR}$ Green I (Molecular Probes, Eugene, OR, USA) (5).
Estimates of DNA concentration are a necessary step in molecular biology. DNA concentration has been estimated in solution either from its absorbance at $260 \mathrm{~nm}$ or fluorescently with the use of specific dyes (6). Fluorescent estimates of DNA concentration offer a higher sensitivity than absorbance measurements so that lower quantities of DNA are needed. The availability of DNA is often a limiting factor, and fluorimetric determinations can be performed with much lower amounts of DNA than absorbance measurements.

In this report, we present the use of an optical thermal cycler, the iQ iCycler $^{\circledR}$ thermal cycler (Bio-Rad Laboratories, Hercules, CA, USA), to estimate the concentration of dsDNA. The $\mathrm{iQ}$ iCycler is designed to obtain fluorescence emission from selected wells during PCR, although the accompanying software is flexible enough to allow for single temperature cycles. It can be used as a high-throughput alternative for estimating DNA concentrations in a 96-well plate format as well as a few tube measurements. This was achieved by designing a simple program for the software accompanying the iQ iCycler. Measurements were performed using a program at a constant temperature for

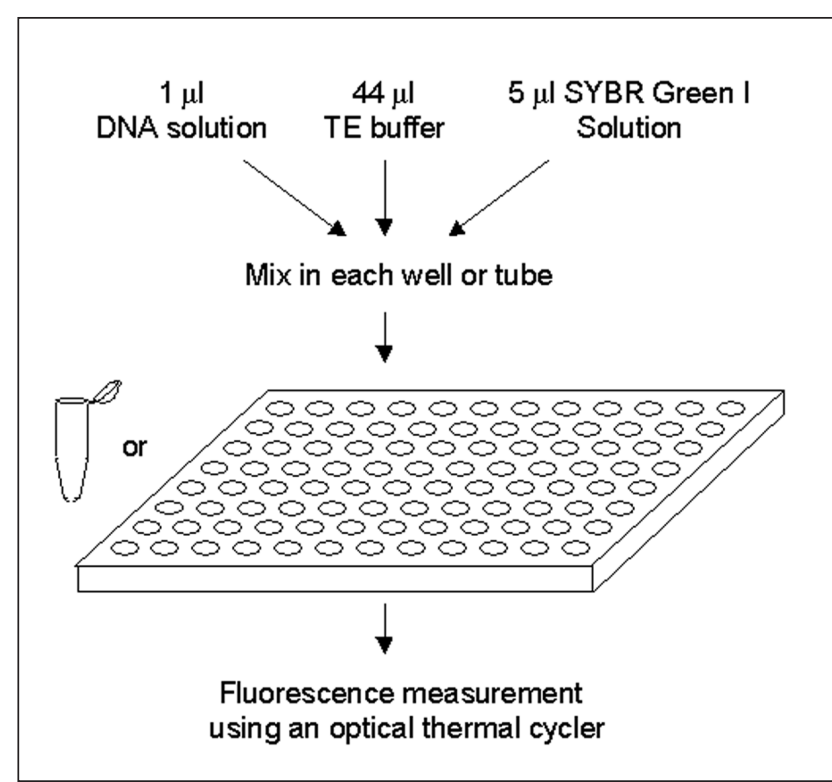

Figure 1. Outline of the protocol presented in this study. The final concentration of SYBR Green I was 1:100 000 .
$1 \mathrm{~min}$ at $25^{\circ} \mathrm{C}$, four cycles with two steps for $12 \mathrm{~s}$ each at $25^{\circ} \mathrm{C}$, obtaining fluorescence measurements during the second steps, and a final $1 \mathrm{~min}$ at $25^{\circ} \mathrm{C}$. These steps resulted in three estimates, which were averaged to obtain the final estimate of dsDNA in the solution. Figure 1 outlines the protocol used for these measurements. The dsDNA was measured in a $50-\mu \mathrm{L}$ solution composed of $1 \mu \mathrm{L}$ DNA solution at the appropriate dilution, $44 \mu \mathrm{L}$ TE buffer, $\mathrm{pH}$ 8.0 (6), and $5 \mu \mathrm{L}$ SYBR Green I (1:100000 final concentration). The use of TE at pH 8.0 maximizes the fluorescence by SYBR Green I because this dye presents its highest fluorescence emission at that $\mathrm{pH}$.

In Figure 2, we present a plot of fluorescence measurements for known DNA concentrations of genomic DNA from E. coli $\mathrm{K} 12$ (CECT 433) and Pseudomonas aeruginosa PAO1 (CECT 4122). Dilutions of the genomic DNA were tested at DNA concentrations ranging from $10^{-8}$ to $0.5 \mu \mathrm{g} / \mu \mathrm{L}$. Linear relationships between fluorescence and DNA concentration occurred at a 5-fold range between 0.1 and $10^{-6} \mu \mathrm{g} / \mu \mathrm{L}$. A regression line for the linear range between DNA concen-

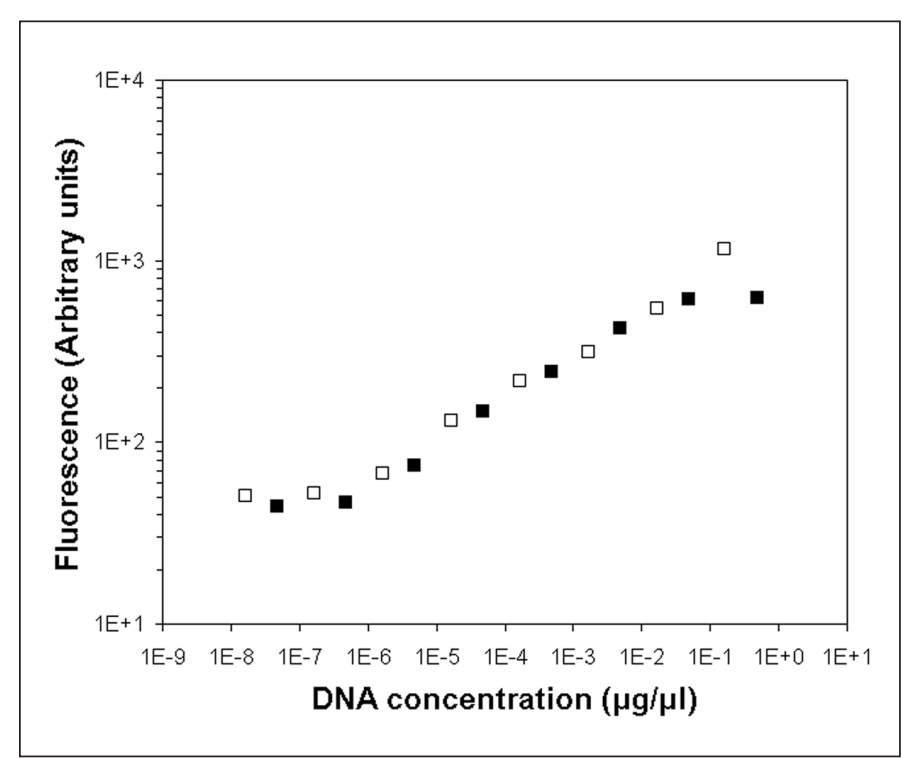

Figure 2. Plot showing fluorescence measurements by an iQ iCycler optical thermal cycler versus DNA concentration in an 8-fold range. The linear relationship was obtained for a 5-fold range between 0.1 and $10^{-6} \mu \mathrm{g} / \mu \mathrm{L}$. Filled squares correspond to genomic DNA from $P$. aeruginosa, and open squares correspond to genomic DNA from $E$. coli. Data points for each replicate were averaged from three measurements. Each data point is the average of two replicates (resulting from three measurements each), and error bars are within the symbols. The coefficient of variation ranged between $0.2 \%$ and $3.5 \%$. 
tration and measured fluorescence of the data shown in Figure 2 was obtained [Bartlet's nonparametric Model II regression analysis (7)] and was represented by the equation: $\log (\mathrm{DNA})=$ $-13.50+4.25 \log$ (Fluorescence). This equation can be used to estimate the concentration of DNA in a solution from fluorescence measured, following the protocol outlined above.

Although estimating DNA concentrations might look trivial, the outcome of several experiments depends on the accurate determinations of DNA in the samples or stock DNA preparations. PCRs can be inhibited by excessive DNA concentrations, and no amplification product will be obtained if DNA is below the amplification threshold. Concentrations of genomic DNA are tricky to estimate because of the viscosity of the concentrated solutions and the unevenness of long DNA in more diluted solutions. This easy and accurate protocol will facilitate the estimation of DNA concentrations. In our laboratory, we perform serial dilutions of genomic DNAs over several orders of magnitude to obtain an accurate estimation of DNA concentrations in stock solutions. The use of a 96-well plate or a multitube approach facilitates this strategy, and the iQ iCycler optical thermal cycler can easily be used for this purpose. The protocol outlined in this study avoids the need to purchase costly fluorescence plate readers, learn how to use an extra apparatus, and simultaneously saves precious bench space.

\section{REFERENCES}

\footnotetext{
1.Morrison, T.B., J.J. Weis, and C.T. Wittwer. 1998. Quantitaion of low copy transcripts by continuous SYBR Green I monitoring during amplification. BioTechniques 24:954-962.

2.Akey, J.M., D. Sosnoski, E. Parra, S. Dios, K. Hiester, B. Su, C. Bonilla, L. Jin, et al. 2001. Melting curve analysis of SNPs (McSNP): a gel-free and inexpensive approach for SNP genotyping. BioTechniques 30:358-367.

3.Ririe, K.M., R.P. Rasmussen, and C.T. Wittwer. 1997. Product differentiation by analysis of DNA melting curves during the polymerase chain reaction. Anal. Biochem. 245:145-160.

4.Gonzalez, J. M. and C. Saiz-Jimenez. 2002. A fluorimetric method for the estimation of $\mathrm{G}+\mathrm{C}$ mol\% content in microorganisms by thermal denaturation temperature. Environ. Microbiol. 4:770-773.

5.Molecular Probes. 1996. SYBR Green I nucleic acid gel stain. Molecular Probes Appli-
}

cation Sheet MP-7567.

6.Sambrook, J., E.F. Fritsch, and T. Maniatis. 1989. Molecular Cloning: A Laboratory Manual. CSH Laboratory Press, Cold Spring Harbor, NY.

7.Sokal, R.R. and F.J. Rohlf. 1981. Biometry,

2nd ed. W.H. Freeman and Co., New York, NY.

We acknowledge support from the Spanish Ministry of Science and Technology through the Ramón y Cajal Program and projects REN2002-00041 and BTE200204492-C02-01. Address correspondence to Dr. Juan M. Gonzalez, IRNAS-CSIC, Apdo 1052, 41080, Sevilla, Spain. e-mail. jmgrau@irnase.csic.es

Received 19 December 2002; accepted 24 January 2003.

\section{Juan M. Gonzalez and Cesareo Saiz-Jimenez \\ Instituto de Recursos Naturales y Agrobiología \\ CSIC, Sevilla, Spain}

\section{Modified 3'-End Amplification PCR for Gene Expression Analysis in Single Cells}

\author{
BioTechniques 34:712-716 (April 2003)
}

For years, gene expression has been widely studied in most fields of biology. It has been used to address molecular mechanisms controlling basic cell functions and their alterations, including cell division, cell differentiation, apoptosis, and various infectious processes. In the vast majority of such studies, carried out by conventional techniques such as Northern blotting or RT-PCR, only the mean gene expression within large populations of cells was analyzed, and cell-to-cell variability was not taken into account. Remarkably, several independent studies using in situ detection techniques have recently pointed to the existence of important intercellular variability in gene expression, both in prokaryotic and mammalian cells. Altogether, the data strongly argue for a stochastic-based process of gene expression (1-4). To further our understanding of cell physiology, there is an increasing need to evaluate intercellular variability of gene expression in a large number of cell types and physiological situations. To this end, several techniques have recently been developed, each with various advantages and limitations (5).

In the course of our work, we used the 3'-end amplification PCR (TPEA$\mathrm{PCR}$ ) procedure, which has proven to be an efficient tool for studying gene expression in various mammalian cell lines at the single-cell level. Although this technique is not quantitative, it allows virtually all the mRNAs sequences present in a single cell to be amplified, including those present at low copy number (6). This RT-PCR relies on two successive amplifications. First, cell lysate-containing mRNAs are reverse-transcribed, followed by a nonspecific amplification, using primers designed to amplify all polyadenylated mRNAs. Second, there is a gene-specific amplification of up to 40 aliquots of the first amplification product. Thus, it is theoretically possible to detect up 